\title{
Perfil de gestantes de alto risco acompanhadas em uma Unidade Básica de
}

\author{
Saúde \\ Profile of high-risk pregnant women followed up in a Basic Health Unit \\ Perfil de gestantes de alto riesgo seguidas en una Unidad Básica de Salud
}

Recebido: 03/08/2021 | Revisado: 13/08/2021 | Aceito: 19/08/2021 | Publicado: 21/08/2021

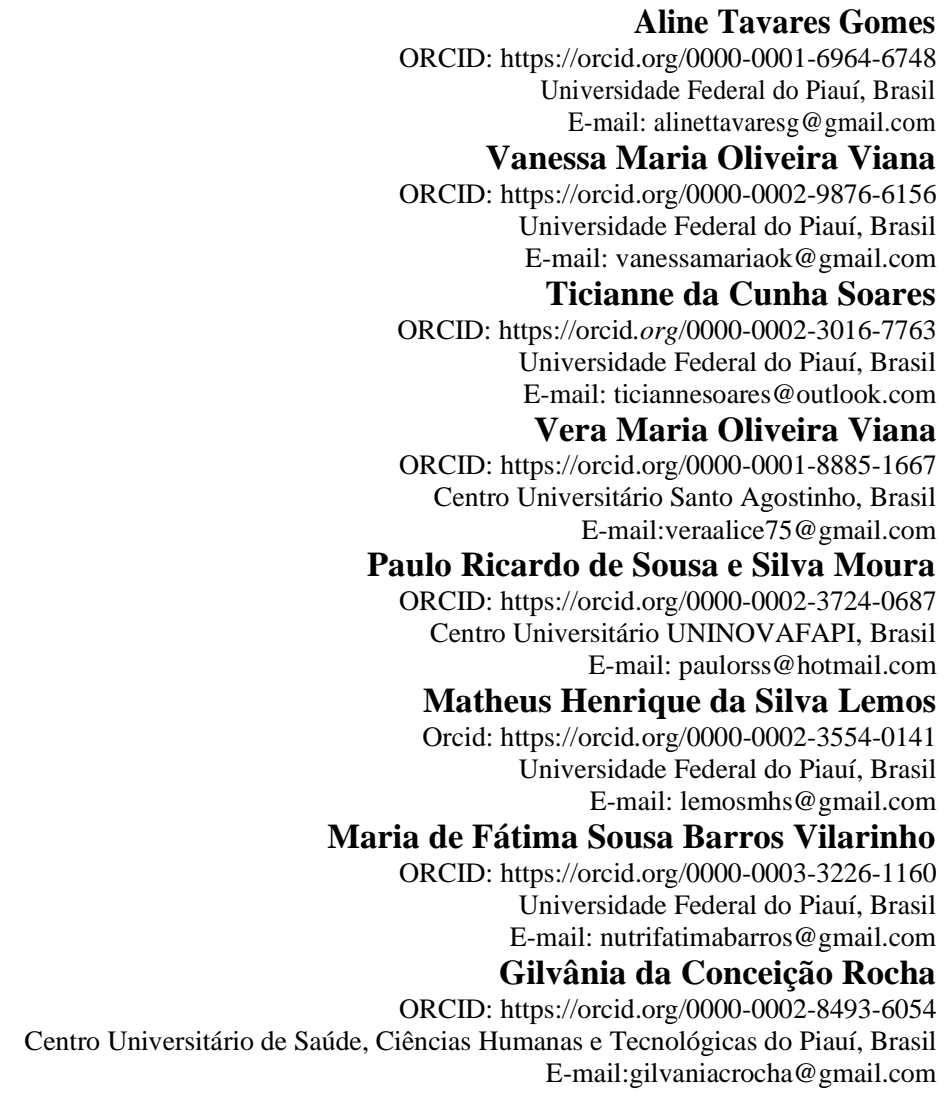

\section{Resumo}

Objetivos: Analisar os casos de gestações de alto risco e suas complicações, além de enfatizar a importância do prénatal e destacar os principais diagnósticos de risco prevalentes em uma Unidade Básica de Saúde (UBS) em um município do estado do Maranhão. Materiais e métodos: Trata-se de um estudo quantitativo do tipo descritivo, desenvolvido em uma UBS do município de Timon, Maranhão, Brasil. Os dados foram coletados nos meses de outubro a novembro de 2019, por meio da análise do prontuário de 20 gestantes. Para a obtenção dos mesmos foi aplicado um instrumento de coleta desenvolvido pelas pesquisadoras exclusivamente para este estudo. A análise foi realizada por meio de estatística descritiva e inferencial. Resultados: O tipo de diagnostico mais frequente entre as gestações de alto risco foi o de Hipertensão Arterial Sistêmica $(95,80 \%)$. Constatou-se que as gestantes com pressão arterial elevada apresentam quatro vezes mais chances de desenvolverem complicações obstétricas ao longo da gestação, culminando em parto cesariano. Conclusão: Conclui-se que é relevante a necessidade de ampliar estudos que deem mais consistência às decisões gerenciais que envolvem o profissional de enfermagem, o serviço de saúde e a saúde da mulher e da criança.

Palavras-chave: Enfermagem; Pré-natal; Gravidez alto risco; Saúde da mulher.

\footnotetext{
Abstract

Objective: The aim of this article was to analyze the cases of high-risk pregnancies and their complications, in addition to emphasizing the importance of prenatal care and highlighting the main risk diagnoses prevalent in a Basic Health Unit (UBS) in a municipality in the state of Maranhão. Materials and method: This is a quantitative, descriptive study, developed in a UBS in the municipality of Timon, Maranhão, Brazil. Data were collected from October to November 2019, through the analysis of the medical records of 20 pregnant women. To obtain them, a collection instrument developed by the researchers exclusively for this study was applied. The analysis was performed
} 
using descriptive and inferential statistics. Results: The most frequent type of diagnosis among high-risk pregnancies was Systemic Arterial Hypertension (95,80\%). It was found that pregnant women with high blood pressure are four times more likely to develop obstetric complications throughout pregnancy, culminating in cesarean delivery. Conclusions: it is concluded that the need to expand studies that give more consistency to management decisions involving the nursing professional, the health service and the health of women and children is relevant.

Keywords: Nursing; Prenatal; High risk pregnancy; Women's health.

\section{Resumen}

Objetivo: analizar los casos de embarazos de alto riesgo y sus complicaciones, además de enfatizar la importancia de la atención prenatal y destacar los principales diagnósticos de riesgo prevalentes en una Unidad Básica de Salud (UBS) en un municipio del estado de Maranhão. Materiales y métodos: Este es un estudio cuantitativo, descriptivo, desarrollado en una UBS en el municipio de Timon, Maranhão, Brasil. Los datos se recopilaron de octubre a noviembre de 2019, a través del análisis de los registros médicos de 20 mujeres embarazadas. Para obtenerlos, se aplicó un instrumento de recolección desarrollado por los investigadores exclusivamente para este estudio. El análisis se realizó mediante estadística descriptiva e inferencial. Resultados: El tipo de diagnóstico más frecuente entre los embarazos de alto riesgo fue la hipertensión arterial sistémica $(95,80 \%)$. Se descubrió que las mujeres embarazadas con presión arterial alta tienen cuatro veces más probabilidades de desarrollar complicaciones obstétricas durante el embarazo, que culminan en un parto por cesárea. Conclusiones: Se concluye que la necesidad de ampliar los estudios que den más coherencia a las decisiones de gestión que involucran al profesional de enfermería, el servicio de salud y la salud de las mujeres y los niños es relevante.

Palabras clave: Enfermería; Cuidado prenatal; Embarazo de alto riesgo; Salud de la mujer.

\section{Introdução}

A gravidez é um período crítico na vida das mulheres visto às diversas mudanças físicas, mentais e sociais as quais estão submetidas. A morbidade materna grave está associada a um aumento do risco de hospitalização e mortalidade no período pós-parto. Dados atuais sugerem que até $88 \%$ das mortes maternas são precedidas por morbidade materna grave. Sendo assim, a redução da mortalidade materna global é estabelecida como uma das metas da agenda 2030 das Nações Unidas nos Objetivos de Desenvolvimento Sustentável (ODS) (James, et al., 2017; Dzakpasu, et al., 2020; Brasil, 2019).

No Brasil, são observados constantes investimentos na busca pela qualificação da atenção à saúde materno-infantil, como a instituição a implantação da Rede Cegonha e da Política Nacional de Atenção Integral à Saúde da Criança (PNAISC), que além de orientarem as práticas a este grupo populacional, organizam os serviços de saúde em nível nacional (Sanine \& Castanheira, 2018).

A redução da mortalidade materna é tema central nas políticas de saúde pautadas na formação de Redes de Atenção à Saúde (RAS), que se constitui como arranjos organizativos de ações e serviços de saúde, de diferentes densidades tecnológicas, que, integradas por meio de sistemas de apoio técnico, logístico e de gestão, buscam garantir a integralidade do cuidado. Os pontos de atenção à saúde são espaços onde se ofertam determinados serviços de saúde de forma singular, cabendo às Unidades Básicas de Saúde (UBS) desempenhar a função de porta de entrada e ordenadora do cuidado em toda a RAS (Brasil, 2012).

Considera-se gestação de alto risco aquela na qual a vida ou a saúde da mãe e/ou do feto e/ou do recém-nascido têm maiores chances de serem atingidas que as da média da população considerada (Brasil, 2012). Em geral, as gestantes de alto risco são encaminhadas para acompanhamento em um serviço especializado, contudo recomenda-se que a gestante mantenha o vínculo com a equipe de atenção primária que iniciou o pré-natal, tal qual a proposta das RAS, incluindo a Rede Cegonha (Brasil, 2016). O acesso oportuno e qualificado aos cuidados pré-natais é um componente essencial para a obtenção de resultados maternos e neonatais favoráveis, visto que permite a realização de classificação de risco e intervenção adequada (Kilpatrick, 2017). Desta maneira, esta pesquisa tem o objetivo de analisar o acompanhamento das gestações de alto risco e suas complicações, além de enfatizar a importância do pré-natal e os principais diagnósticos prevalentes em uma Unidade Básica de Saúde (UBS) em um município do estado do Maranhão/Brasil. 


\section{Metodologia}

Trata-se de um estudo transversal e descritivo de abordagem quantitativa, desenvolvido em uma UBS do município de Timon, localizado no estado do Maranhão/ Brasil. A população estudada foi recrutada de forma censitária, sendo consideradas para o estudo todas as gestantes classificadas como de alto risco em acompanhamento pela Equipe de Estratégia de Saúde da Família (ESF) da UBS pesquisada. Para efeito deste estudo considerou-se gestantes de alto risco aquelas que possuíam uma patologia prévia ou desencadeada durante o processo gravídico, de acordo com as recomendações do Ministério da Saúde (Brasil, 2012).

A pesquisa quantitativa trata da quantificação e análise de variáveis para a obtenção de resultados. Envolve a utilização e análise de dados numéricos usando técnicas estatísticas específicas para responder a perguntas como quem, quanto, o quê, onde, quando, quantos e como. Também descreve os métodos de explicação de um problema ou fenômeno por meio da coleta de dados na forma numérica (Apuke, 2017).

Nos métodos quantitativos, faz-se a coleta de dados quantitativos ou numéricos por meio do uso de medições de grandezas e obtém-se por meio da metrologia, números com suas respectivas unidades. Estes métodos geram conjuntos ou massas de dados que podem ser analisados por meio de técnicas matemáticas como é o caso das porcentagens, estatísticas e probabilidades, métodos numéricos, métodos analíticos e geração de equações e/ou fórmulas matemáticas aplicáveis a algum processo (Estrela, 2018).

A amostra do estudo foi constituída por vinte gestantes. Contabilizou-se o número de gestantes dos dois últimos anos, considerando o período de 2018 a 2019, e avaliado o grupo e a patologia clínica que a gestante apresentava de acordo com o Código Internacional de Doenças - CID. Com a análise dos dados foi possível realizar a categorização dos principais agravos a saúde na gestação.

Os critérios de inclusão deste estudo foram: mulheres com doenças crônicas prévias à gestação, mulheres com gestação anterior de alto risco, e mulheres que identificaram, no curso do processo gravídico, uma condição ou doença de risco materno e/ou fetal. Foram excluídas da pesquisa as mulheres que sofreram abortos espontâneos e as gestações que obtiveram como desfecho o óbito neonatal.

Os dados foram coletados nos meses de setembro a novembro de 2019 por meio de um instrumento específico criado pelas autoras deste estudo, utilizando as variáveis: sexo, idade, estado civil, tempo de gestação (expresso em semanas), agravos de saúde (atual ou posterior a gravidez), diagnóstico de risco e histórico obstétrico.

Após a obtenção dos dados, estes foram digitados e armazenados em uma planilha eletrônica (Excel - Microsoft Office 2013) e analisados utilizando os softwares Statistical Package for the Social Sciences - SPSS 20.0 e BioEstat 5.0. Para descrever os dados coletados foram calculadas as frequências absolutas e percentuais.

Com o intuito de verificar a diferença entre as frequências das categorias de uma mesma variável empregou-se o teste de Qui-quadrado de aderência para proporções esperadas iguais. O teste do Qui-quadrado de independência foi utilizado para verificar a associação entre o tempo gestacional e a ocorrência de complicações obstétricas.

Ressalta-se que a pesquisa obedeceu aos princípios éticos norteadores de pesquisas envolvendo seres humanos, dispostos na Resolução no 466/12 do Conselho Nacional de Saúde, sendo submetida e aprovada pelo Comitê de Ética em Pesquisa da Universidade Federal do Piauí (Brasil, 2012).

\section{Resultados e Discussão}

A gravidez desenvolve-se em um contexto social e cultural que influencia e determina a sua evolução e a sua trajetória. Assim, é importante considerar fatores como a história pessoal da gestante e seu passado obstétrico, bem como a 
conjuntura sociodemográfica e econômica a qual ela está inserida para assim conhecer os fatores de risco que possam interferir na saúde materno-fetal e direcionar a assistência ao binômio mãe-filho (Rodrigues, et al., 2017).

Ressalta-se que condições de risco requerem consultas obstétricas de forma frequente, assim é imprescindível a realização do pré-natal de alto risco de forma compartilhada entre a Atenção Primária em Saúde (APS) e a atenção de nível secundário, realizada em hospitais ou em ambulatórios específicos. O seguimento desta recomendação visa à garantia da qualidade da atenção à saúde da mãe e da criança, bem como o parto seguro e o desenvolvimento saudável (Paiva, Nunes, Moreira, \& Ferreira, 2019).

A partir da análise dos 20 prontuários deste estudo, verificou-se que a maioria das gestantes estudadas $(60,00 \%)$ tinha idade entre 15 e 30 anos $(\mathrm{n}=12), 40,00 \%(\mathrm{n}=8)$ destas mulheres não possuíam vínculo empregatício, 80,00\% (n=16) possuíam o segundo grau completo, e $85,00 \%(n=17)$ eram casadas e/ou possuíam uma união estável. Quanto a cor de pele autorreferida, $80,00 \%(n=16)$ se declararam como de cor parda (Tabela 1$)$.

Tabela 1 - Características demográficas das gestantes estratificadas como pré-natal de alto risco em uma UBS, Timon, Maranhão, Brasil, 2019.

\begin{tabular}{|c|c|c|}
\hline Variáveis & $\mathbf{N}$ & $\%$ \\
\hline \multicolumn{3}{|l|}{ Idade } \\
\hline Entre $15-30$ anos & 12 & $60,00 \%$ \\
\hline Acima de 30 anos & 8 & $40,00 \%$ \\
\hline \multicolumn{3}{|l|}{ Profissão } \\
\hline Desempregadas & 8 & $40,00 \%$ \\
\hline Comerciário & 6 & $30,00 \%$ \\
\hline Outros & 6 & $30,00 \%$ \\
\hline \multicolumn{3}{|l|}{ Escolaridade } \\
\hline 1 grau & 4 & $20,00 \%$ \\
\hline 2 grau & 16 & $80,00 \%$ \\
\hline \multicolumn{3}{|l|}{ Estado Civil } \\
\hline Casada & 4 & $20,00 \%$ \\
\hline União Estável & 13 & $65,00 \%$ \\
\hline Solteira & 2 & $10,00 \%$ \\
\hline Separada & 1 & $5,00 \%$ \\
\hline \multicolumn{3}{|l|}{ Etnia } \\
\hline Parda & 16 & $80,00 \%$ \\
\hline Negra & 2 & $10,00 \%$ \\
\hline Branca & 2 & $10,00 \%$ \\
\hline TOTAL & 20 & 100,00 \\
\hline
\end{tabular}

Fonte: Autores (2021).

Esses dados corroboram com outras pesquisas, a exemplo das realizadas por Brito, Lopes e Barros (Brito, Lopes \& Barros, 2020), que apresentaram resultados semelhantes quanto aos aspectos demográficos. Sabe-se também que a interferência da baixa condição socioeconômica na autonomia da mulher evidencia um conflito diante da gravidez devido à ausência de estabilidade financeira, dificultando o acesso a informações e aos meios que permitem a tomada de decisão (Vitória \& Moreira, 2017).

Dentre as gestantes analisadas, 14 mulheres (70,00\%) possuíam antecedentes familiares de patologias consideradas como de risco, sendo mais prevalente a Hipertensão Arterial Sistêmica (HAS) verificada em 16 (80,00\%) das mulheres participantes da pesquisa, seguida pela Diabetes Mellitus (DM) (45,00\%) (n=9) (Tabela 2). 
Tabela 2 - Antecedentes familiares das gestantes estratificadas como gestação de alto risco na UBS. Timon, Maranhão, Brasil, 2019.

\begin{tabular}{lccc}
\hline \multicolumn{1}{c}{ Variáveis } & $\begin{array}{c}\text { Sim } \\
\text { N (\%) }\end{array}$ & $\begin{array}{c}\text { Não } \\
\text { N (\%) }\end{array}$ & TOTAL \\
\hline \multicolumn{1}{c}{ HAS } & $16(80,00 \%)$ & $4(20,00 \%)$ & \\
\multicolumn{1}{c|}{ DM } & $9(45,00 \%)$ & $11(55,00 \%)$ & $\mathbf{2 0}(\mathbf{1 0 0 \% )}$ \\
Infecções do trato urinário & $14(70,00 \%)$ & $6(30,00 \%)$ & $15(75,00 \%)$ \\
Gemelaridade & $5(25,00 \%)$ & $12(60,00 \%)$ & \\
\hline Outros & $8(40,00 \%)$ & \\
\hline
\end{tabular}

Fonte: Autores (2021).

Importa ressaltar o caráter hereditário e a forte relação da HAS e do DM com altas taxas de mortalidade e mobimortalidade, principais patologias identificadas nos antecedentes familiares das gestantes participantes do presente estudo (15). Estudo conduzido por Meazaw et al aponta que o risco de desenvolver distúrbios hipertensivos na gestação é maior entre as mulheres com histórico de HAS (seja ela própria ou sua família) (Meazaw, Chojenta, Muluneh, \& Loxton, 2020; Soares, et al., 2019).

Quanto aos antecedentes pessoais obstétricos, verificou-se que 90,00\% ( $\mathrm{n}=18)$ das gestantes eram multíparas, e 11 destas mulheres se classificaram como gesta $2(55,00 \%)$, isto é, tiveram duas gestações durante a vida. Resultado semelhante foi encontrado nas regiões de Santarém, no estado do Pará/ Brasil, e na região central do Rio Grande do Sul/Brasil, onde a maioria das mulheres acompanhadas no pré-natal de alto risco era multípara (Xavier, Jannotti, Silva, \& Martins, 2013; Jantsch, et al., 2017; Soares, et al., 2019).

O tipo de parto mais prevalente no estudo foi o parto cesariano, sendo este o desfecho da gravidez de 15 gestantes, correspondendo a 75,00\%. (Tabela 3). Ressalta-se que o parto cesariano está relacionado com maior morbimortalidade materna e pode trazer complicações nas gestações subsequentes (McClelland, et al., 2017).

Tabela 3 - Antecedentes pessoais obstétricos das gestantes estratificadas como gestação de alto risco na UBS e o tipo de parto resultante da gestação atual. Timon, Maranhão, Brasil, 2019.

\begin{tabular}{|clr|}
\hline Variáveis & N & $(\boldsymbol{\%})$ \\
\hline Nulípara & 2 & $10,00 \%$ \\
\hline Multípara & 18 & $90,00 \%$ \\
\hline Número de gestações & & \\
\hline Gesta 1 & 2 & $10,00 \%$ \\
\hline Gesta 2 & 11 & $55,00 \%$ \\
\hline Gesta 3 ou mais & 7 & $35,00 \%$ \\
\hline Tipos de parto & & \\
\hline Cesariana & 15 & $75,00 \%$ \\
\hline Normal & 5 & $25,00 \%$ \\
\hline Total & 20 & 100 \\
\hline
\end{tabular}

Fonte: Autores (2021).

O principal motivo pelo qual a gestante classificou-se como alto risco no pré-natal foi o acometimento da HAS, 
diagnosticada em 60,00\% (n=12) das gestantes, seguida da obesidade com 45,00\% (n=9), e da Infecção do Trato Urinário (ITU), relatada por 40,00\% das participantes $(n=8)$. Vale destacar que este estudo considerou uma ou mais patologia manifestada pelas participantes, sendo descrito nas tabelas todas as condições clínicas citadas pelas mulheres durante a gravidez (Tabela 4).

Tabela 4 - Doenças prevalentes nas mulheres estratificadas como gestação de alto risco na UBS. Timon, Maranhão, Brasil, 2019.

\begin{tabular}{|c|c|c|c|c|c|}
\hline Diagnósticos & & $\mathbf{N}(\%)$ & Média & Min-Máx & $\begin{array}{l}\text { Desvio } \\
\text { Padrão }\end{array}$ \\
\hline HAS & & $12(0,60 \%)$ & & & \\
\hline DM & & $6(0,30 \%)$ & & & \\
\hline Obesidade & & $9(0,45 \%)$ & & & \\
\hline Cardiopatias & & $3(0,15 \%)$ & & & \\
\hline $\begin{array}{l}\text { Infeções no } \\
\text { Urinario }\end{array}$ & Trato & $8(0,40 \%)$ & 6,7 & $1,0-40,0$ & 7,2 \\
\hline $\begin{array}{ll}\text { Infecções no } \\
\text { Respiratorio }\end{array}$ & Trato & $5(0,25 \%)$ & & & \\
\hline HIV & & $2(0,10 \%)$ & & & \\
\hline Sífilis & & $1(0,05 \%)$ & & & \\
\hline TOTAL & & $20(100 \%)$ & & & \\
\hline
\end{tabular}

Fonte: Autores (2021).

Os distúrbios hipertensivos que complicam a gravidez, especialmente a hipertensão gestacional, é uma das complicações comuns que são exclusivas da gravidez, e sua patogênese específica atualmente não é clara, o que pode levar a uma variedade de resultados adversos da gravidez e prejudicar seriamente a saúde de mães e crianças (Osibogun, Ogunmoroti, \& Michos, 2020).

O sobrepeso e a obesidade são agora as condições de alto risco mais comuns na gravidez e aumentam o risco de resultados adversos durante a gravidez, o parto e o pós-parto. É importante ressaltar que o ganho de peso gestacional em excesso é altamente preditivo de retenção de peso materno pós-parto e risco de sobrepeso e obesidade em mães e seus filhos mais tarde na vida. Isso torna a gravidez uma janela de oportunidade única na luta contra a obesidade ao longo da vida (Blau, \& Hormes, 2020).

Já as infecções do trato urinário (ITUs) são frequentemente encontradas em mulheres grávidas. Portanto, é crucial que os prestadores de cuidados obstétricos conheçam os achados normais do trato urinário, a avaliação das anormalidades e o tratamento da doença (Habak \& Griggs, 2021).

Para a análise de correlações de variáveis foi aplicada regressão logística com as variáveis descritas nas tabelas acima e o diagnóstico de HAS, patologia de risco mais encontrada no presente artigo, e que apresentou um valor de p significativo na regressão logística binária (Tabela 5).

Nas análises do presente estudo houve associação de variáveis com o diagnóstico de HAS, apresentando fator estatístico significativo de quatro vezes mais chances de complicações no parto de gestantes classificadas como de risco, corroborando com estudo desenvolvido por Silva, et al., 2019. 
Tabela 5 - Regressão logística dos dados sociodemográficos em relação aos antecedentes obstétricos, tipo de parto e as doenças prevalentes na UBS. Timon (MA), $(\mathrm{N}=20)$.

\begin{tabular}{|c|c|c|c|c|c|}
\hline & \multirow[t]{2}{*}{ B } & \multirow[t]{2}{*}{ P-valor } & \multirow[t]{2}{*}{ OR } & \multicolumn{2}{|c|}{ 95\% C.I. para OR } \\
\hline & & & & & Superior \\
\hline Idade & 0,024 & 0,186 & 1,024 & 0,989 & 1,060 \\
\hline \multicolumn{6}{|l|}{ Estado Civil } \\
\hline Solteiro & & & 1 & & \\
\hline Casado & 0,103 & 0,777 & 1,109 & 0,543 & 2,262 \\
\hline Divorciado & 1,864 & 0,116 & 6,451 & 0,631 & 65,950 \\
\hline \multicolumn{6}{|c|}{ Antecedentes Obstétricos } \\
\hline Nulípara & & & 1 & & \\
\hline Multípara & 1,281 & 0,059 & 3,599 & 0,954 & 13,573 \\
\hline \multicolumn{6}{|c|}{ Tipos de parto } \\
\hline Cesariano & & & 1,000 & & \\
\hline Normal & 2,201 & 0,052 & 9,032 & 0,979 & 83,329 \\
\hline \multicolumn{6}{|c|}{ Doenças prevalentes } \\
\hline DM & & & 1,000 & & \\
\hline HAS & $-2,000$ & 0,148 & 0,135 & 0,009 & 2,035 \\
\hline
\end{tabular}

*Teste de associação qui-quadrado $\left(\mathrm{X}^{2}\right)$. Fonte: Autores (2021).

A pré-eclâmpsia é descrita como fator de risco para morbidade e mortalidade das mulheres mesmo após o parto. Estudo realizado por Chourdakis et al aponta associação entre a pré-eclâmpsia e doença cardiovascular mais tarde na vida, muito anos após a manifestação desse distúrbio hipertensivo relacionado à gravidez, o que requer diagnóstico precoce e acompanhamento qualificado (Chourdakis, Oikonomou, Fouzas, Hahalis, \& Karatza, 2021). Murray et al. também demonstrou que mulheres com distúrbios hipertensivos na gestação tem risco aumentado de praticamente todas as complicações pós-parto, incluindo aquelas não relacionadas à gestação (Murray Hor witz, Rodriguez, Dissanayake, Carmichael, \& Snowden, Jonathan, 2021).

Nesta pesquisa não foi verificada associação estatística $(\mathrm{p}>0,05)$ para escolaridade e cor da pele, estado civil ou antecedentes obstétricos da gestante. Contudo, outros estudos realizados no país destacam relevantes relações entre desigualdades raciais e sociodemográficas e a assistência ao pré-natal e ao parto, sendo importante o estímulo à reflexão e desenvolvimento de outros artigos com enfoque central nessas variáveis.

\section{Conclusão}

O presente trabalho evidenciou os principais diagnósticos de gestações de alto risco detectados em uma Unidade Básica de Saúde, bem como investigou os principais condicionantes e determinantes de saúde que prevalecem nesses diagnósticos. Dessa forma, buscou por meio dos resultados encontrados possíveis correlações dos problemas obstétricos com a situação social e comportamental das gestantes portadoras dos diagnósticos de alto risco, facilitando e incentivando novos estudos e reflexões sobre o tema.

Entende-se que o debate acerca do pré-natal de alto risco e seu acompanhamento na APS contribui para o aprofundamento da discussão sobre esta temática, de modo a agregar novos conhecimentos que visam contribuir com a implementação de ações voltadas à saúde da mulher por meio de estratégias que possibilitem a prevenção de desfechos desfavoráveis em decorrência de complicações obstétricas.

Assim, o estudo além de relevante para a saúde pública, desperta a atenção da enfermagem, especialmente do campo da obstetrícia, na perspectiva da necessidade de investigação e intervenção no que se refere à assistência e promoção da saúde, 
podendo subsidiar a adoção de estratégias preventivas relacionadas à saúde da mulher.

Ressalta-se ainda que novos estudos envolvendo tal temática são imprescendíveis para compreensão e intervenção em fenômenos obstétricos desfavoráveis. Sugere-se que estudos com amostras populacionais maiores sejam realizados, visto a limitação deste fator neste artigo; bem como o desenvolvimento de pesquisas que deêm mais consistência às decisões gerenciais que envolvem o profissional de enfermagem, o serviço de saúde e a saúde da mulher e da criança.

\section{Referências}

Apuke, O. D. (2017). Quantitative Research Methods: A Synopsis Approach. Arabian J Bus Manag Review (kuwait Chapter), 6(10): 40-47.

Blau, L. E., \& Hormes, J. M. (2020). Preventing Excess Gestational Weight Gain and Obesity in Pregnancy: the Potential of Targeting Psychological Mechanisms. Curr Obes Rep, 9(3): 522-529.

Brasil. Ministério da Saúde (2012). Gestação de alto risco: manual técnico. Secretaria de Atenção à Saúde. Departamento de Ações Programáticas Estratégicas. (5a ed). Ministério da Saúde.

Brasil. Ministério da saúde (2012). Resolução $n^{\circ} 466$, de 12 de dezembro de 2012. Aprova as diretrizes e normas regulamentadoras de pesquisas envolvendo seres humanos. Conselho nacional de saúde. Diário Oficial da República Federativa do Brasil.

Brasil. Ministério da Saúde/ Instituto Sírio-Libanês de Ensino e Pesquisa (2016). Protocolo da Atenção Básica: Saúde das Mulheres. Secretaria de Atenção à Saúde Departamento de Atenção Básica, Ministério da saúde.

Brito, L. S. D. A., Lopes, L. F., \& Barros, L. (2020). Perfil epidemiológico de gestantes de alto risco e o acompanhamento realizado por enfermeiros na regional ilha do bananal no estado do Tocantins. Science \& health, 8(1), 66-77.

Bundy, J. D., Li, C., Stuchlik, P., Bu, X., Kelly, T. N., Mills, K. T., He, H., Chen, J., Whelton, P. K., \& He, J. (2017). Systolic blood pressure reduction and risk of cardiovascular disease and mortality: a systematic review and network meta-analysis. JAMA Cardiol, 2(7):775-781.

Chourdakis, E., Oikonomou, N., Fouzas, S.; Hahalis, G., \& Karatza, A. A. (2021). Preeclampsia Emerging as a Risk Factor of Cardiovascular Disease in Women. High Blood Press Cardiovasc Prev, 28(6): 103-114.

Dzakpasu, S., Deb-Rinker, P., Arbour, L., Darling, E. K., Kramer, M. S., Liu, S., Luo, W., Murphy, P. A., Nelson, C., Ray, J. G., Scott, H., VandenHof, M.; \& Joseph, K. S. (2020). Severe maternal morbidity surveillance: monitoring pregnant women at high risk of prolonged hospitalization and death. Paediatr Perinat Epidemiol, 34(4),427-439.

Estrela, C. (2018). Metodologia Científica: Ciência, Ensino, Pesquisa. Editora Artes Médicas. UFSM.

Habak, P. J., \& Griggs, Jr.R. P. (2021). Urinary Tract Infection In Pregnancy. In: StatPearls. Treasure Island (FL): StatPearls Publishing..

James, D. K., Steer, P. J., Weiner, C. P., Gonik, B., Crowther, C. A., \& Robson, S. C. (2017). High risk pregnancy (5ºd). Cambridge: Cambridge University press.

Jantsch, P. F., Carreno, I., Pozzobon, A., Adami, F. S., Leal, C. D. S., Mathias, T. C. D. S., Ludwing, A. S., \& Bergo P. H. F (2017). Principais características das gestantes de alto risco da região central do Rio Grande do Sul. Revista Destaques Acadêmicos, 9 (3), 1-11.

Kilpatrick, S. J (2017). American Academy of Pediatrics; American College of Obstetricians and Gynecologists. Guidelines for Perinatal Care (8a ed). Elk Grove Village.

McClelland, S., Gorfinkle, N., Arslan, A. A., Benedetto-Anzai, M. T., Cheon, T., \& Anzai, Y. (2017). Factors associated with cesarean delivery rates: a singleinstitution experience. Matern Health Neonatol and Perinatol, 3(8), 1-8.

Meazaw, M. W., Chojenta, C., Muluneh, M. D., \& Loxton, D. (2020). Factors associated with hypertensive disorders of pregnancy in sub-Saharan Africa: A systematic and meta-analysis. PLoS One, 15(8):e0237476.

Murray Horwitz, M. E., Rodriguez, M. I., Dissanayake, M., Carmichael, SL., \& Snowden, Jonathan, M. S. (2021). Postpartum health risks among women with hypertensive disorders of pregnancy, California 2008-2012. Journal of Hypertension, 39 (5): 1009-1017.

ONU. (2019). Objetivos de desenvolvimento sustentável (ODS). Organização das Nações Unidas (ONU). https://www.paho.org/bra/index.php?option=co m_content\&view=article\&id=5849:objetivos-de-desenvolvimento-sustentavel\&Itemid=875.

Osibogun, O., Ogunmoroti, O., \& Michos, E. D. (2020). Polycystic ovary syndrome and cardiometabolic risk: Opportunities for cardiovascular disease prevention. Trends Cardiovasc Med, 30 (7): 399-404.

Paiva, D. S. D. B. S., Nunes, H. H. D. M., Moreira, S. F. D. S., \& Ferreira, M. G. S. (2019). Pré-natal de alto risco em um serviço de referência: perfil sociodemográfico e clínico. Revista Eletrônica Acervo Saúde, 11(2), e136.

Rodrigues, A. R. G., Dantas, S. L. D. C., Pereira, A. N. M., Silveira, M. A. M. D., \& Rodrigues, D. P. (2017). Gravidez de alto risco: análise dos determinantes de saúde. Revista Sanare, 16(1), 23-28.

Sanine, P. R., \& Castanheira, E. R. L. (2018). Explorando nexos entre a construção social da criança e as práticas de saúde. Hist Ciênc Saúde-Manguinhos, 25(1):199-215. 
Research, Society and Development, v. 10, n. 11, e02101119038, 2021

(CC BY 4.0) | ISSN 2525-3409 | DOI: http://dx.doi.org/10.33448/rsd-v10i11.19038

Santos, L. (2017). Região de saúde e suas redes de atenção: modelo organizativo-sistêmico do SUS. Ciênc. Saúde Colet., 22(4): 1281-1289.

Silva, J. D. C., Carvalho Filha, F. S. S., Silva, M. V. D. R. S., Silva, E. A. C., \& Santos, J. C. (2019). Pré-Natal de alto risco: dados sociodemográficos e intercorrências durante a gravidez. Revista Eletrônica Acervo Saúde; 23(451), 1-8.

Soares, T. C., Santana, L. C. B., Soares, T. C., Ferreira, J. C. S. C., Luz, A. C., Vilarinho, M. F. S. B., Leal, J. D. V., Oliveira, V. A., Almeida, J. R., Araújo, V. S., \& Andrade, R. F. (2019). Fatores de risco relacionados a pré-eclâmpsia: uma revisão integrativa da literatura. Revista Eletrônica Acervo Saúde, 20(e437): $1-8$.

Vitória, T. O. D. O., \& Moreira, R. D. C. R. (2017). Acessibilidade de gestantes ao pré-natal de alto risco. Rev. Saúde Col. UEFS, 7(3): 45-51.

Xavier, R. B., Jannotti, C. B., Silva, K. S. D., \& Martins, A. D. C. (2013). Risco reprodutivo e renda familiar: análise do perfil de gestantes. Ciênc Saúde Coletiva, 18(4):1161-1171. 